\title{
EMISIÓN DIFUSA DE HIDRÓGENO EN EL VOLCÁN POÁS, COSTA RICA
}

\author{
Gladys V. Melián ${ }^{1 *}$, Inés Galindo ${ }^{1}$, Nemesio M. Pérez ${ }^{1}$, Pedro A. Hernández ${ }^{1}$, \\ José M. L. Salazar ${ }^{1}$, Mario Fernández ${ }^{2}$, Carlos Ramírez², Raúl Mora ${ }^{3}$ \\ \& Guillermo E. Alvarado ${ }^{3}$ \\ ${ }^{1}$ División de Medio Ambiente, Instituto Tecnológico y de Energías Renovables \\ (ITER), 38611 Granadilla, S/C de Tenerife, Islas Canarias, España \\ ${ }^{2}$ Centro de Investigaciones Geofísicas, Universidad de Costa Rica, Costa Rica \\ ${ }^{3}$ Escuela Centroamericana de Geología, Universidad de Costa Rica, Costa Rica \\ * Autora para contacto: gladys@iter.rcanaria.es
}

(Recibido 31/08/03; aceptado 04/09/03)

\begin{abstract}
We report the results of the study of diffuse $\mathrm{H}_{2}$ emission from Poás volcano, Costa Rica. Three surveys were carried out in 2000, 2001 and 2002, to investigate soil $\mathrm{H}_{2}$ distribution in and around Poás summit crater and to evaluate diffuse $\mathrm{H}_{2}$ emission as a potential geochemical tool for the Poás volcano monitoring program. Soil $\mathrm{H}_{2}$ contents showed a wide range of concentrations from 0.4 to 7,059 ppmV. Most of the study area showed low levels of $\mathrm{H}_{2}$ in the soil atmosphere, and the highest values of soil gas $\mathrm{H}_{2}$ were just observed inside the summit crater of Poás volcano for the 2001 and 2002 surveys. These surface geochemical changes coincide with an increase of the fumarolic activity as well as soil temperature rose inside the crater during this study. The results here reported suggest changes in the Poás volcanic-hydrothermal system, therefore monitoring diffuse $\mathrm{H}_{2}$ emission could be useful for the volcanic surveillance of Poás volcano.
\end{abstract}

Keywords: Poás volcano, hydrogen, diffuse emission, volcano surveillance, main crater, fumaroles, volcanichydrothermal system.

RESUMEN: Se presentan los resultados obtenidos del estudio de las emanaciones difusas de $\mathrm{H}_{2}$ en el volcán Poás, Costa Rica. Este estudio ha implicado la realización de tres campañas en los años 2000, 2001 y 2002, con la finalidad de investigar la distribución espacial de la concentración de $\mathrm{H}_{2}$ en el gas del suelo y evaluar la desgasificación difusa de esta especie como una herramienta geoquímica para el programa de vigilancia volcánica del Poás. Las concentraciones de $\mathrm{H}_{2}$ en el gas del suelo medidas presentan un amplio rango de valores que van desde 0,4 a 7059 ppmV. La mayor parte del área de estudio reflejó contenidos relativamente bajos de $\mathrm{H}_{2}$ en el gas del suelo, mientras que las concentraciones más altas se midieron en el interior del cráter principal durante los años 2001 y 2002. Desde la campaña del 2001 se ha observado un aumento relativo del contenido de $\mathrm{H}_{2}$ en el gas del suelo del interior del cráter principal, coincidiendo en el tiempo con un incremento de la actividad fumarólica y de la temperatura en los suelos. Estos resultados pueden indicar la existencia de cambios en el sistema volcánico-hidrotermal del Poás, por lo que el monitoreo de las emanaciones difusas de $\mathrm{H}_{2}$ podría ser útil para mejorar el programa de vigilancia volcánica.

Palabras clave: Volcán Poás, hidrógeno, emisión difusa, vigilancia volcánica, cráter principal, fumarolas, sistema volcánico-hidrotermal. 


\section{INTRODUCCIÓN}

Desde hace tiempo es conocido que los gases disueltos en el magma proporcionan la fuerza motriz de las erupciones volcánicas, pero solo el reciente desarrollo de nuevas técnicas ha permitido la realización de medidas rutinarias de los diferentes tipos de gases que son emitidos hacia la atmósfera por los volcanes (Hirabayashi et al., 1986; Sato, 1988; Sano et al., 1991; Casadevall et al., 1994; Giggenbach, 1996; McGee \& Gerlach, 1998; Hernández et al., 2001a). Las primeras manifestaciones que se observan en un volcán activo, son generalmente las emisiones fumarólicas, ricas en vapor de agua y azufre, aunque también se escapan a la atmósfera una importante cantidad de gases a través de otras manifestaciones visibles, como hervideros y penachos volcánicos, así como a través del suelos en forma difusa y dispersa, emisiones conocidas como "no visibles".

La mayor parte de los estudios sobre emisión difusa de gases en sistemas volcánicos se han centrado en el dióxido de carbono por su baja solubilidad en los fundidos silicatados y su condición de componente mayoritario en los gases volcánicos (Gerlach \& Graeber, 1985; Stolper \& Holloway, 1988, Pan et al., 1991). Por el contrario, los estudios realizados de emisiones difusas de las especies volátiles trazas en gases volcánicos, tales como el helio y el hidrógeno, son escasos (Sato \& McGee, 1981; Lombardi \& Reimer, 1990; Hernández et al., 2000). Por otro lado, el estudio de los niveles de flujo y concentración de los gases en el suelo y la evaluación de la evolución temporal de las anomalías nos proporciona una importante información sobre el estado de actividad del sistema volcánico (Sato \& McGee, 1981; Lombardi \& Reimer, 1990; Allard et al., 1991; Pérez et al., 1996; Hernández et al., 2001a, 2001b, Salazar et al., 2001). El hidrógeno es de gran interés por estar presente en la mayoría de las emisiones volcánicas (White \& Waring, 1963). Su alta movilidad dentro de la corteza terrestre en comparación con otras especies volátiles y su característica de gas ligero, lo convierten en uno de los mejores indicadores geoquímicos de la actividad magmática en profundidad (Sato, 1988).
El volcán Poás es un estratovolcán andesítico-basáltico de $2708 \mathrm{~m}$ de altitud y es uno de los 41 volcanes históricamente activos de la cadena volcánica de América Central (Simkin \& Siebert, 1994). El Poás se encuentra 35 km al noroeste de San José y está constituido por tres cráteres alineados en dirección N-S (Fig. 1). El cráter principal, de forma subcónica irregular y con un diámetro de $1,3 \mathrm{~km}$ y $320 \mathrm{~m}$ de profundidad, presenta dos particularidades: (a) una laguna áci$\mathrm{da}(\mathrm{pH}=0)$ de unos $300 \mathrm{~m}$ de diámetro, con una temperatura entre 30 y $60^{\circ} \mathrm{C}$, cuyo volumen, temperatura y propiedades químicas varían con el régimen pluviométrico y la actividad volcánica (Brown et al., 1989; Rowe et al., 1992a, 1992b) y (b) un domo situado al sureste de la laguna. Este domo se eleva $40 \mathrm{~m}$ sobre la laguna y se formó durante la erupción freatomagmática y magmática de 1953-1954 (Casertano et al., 1985, 1987; Rowe et al., 1992a). En el flanco noroeste del domo se localizan numerosas fumarolas activas de temperatura e intensidad variable. Los gases emitidos por estas fumarolas unidos a los aerosoles procedentes de la laguna son los responsables de la pluma característica del volcán.

Al norte del cráter principal se encuentra el cono Von Frantzius (2639 m s.n.m.), cubierto de vegetación, con forma de herradura y altamente erosionado, actualmente inactivo. El otro cono presente en la estructura es el denominado

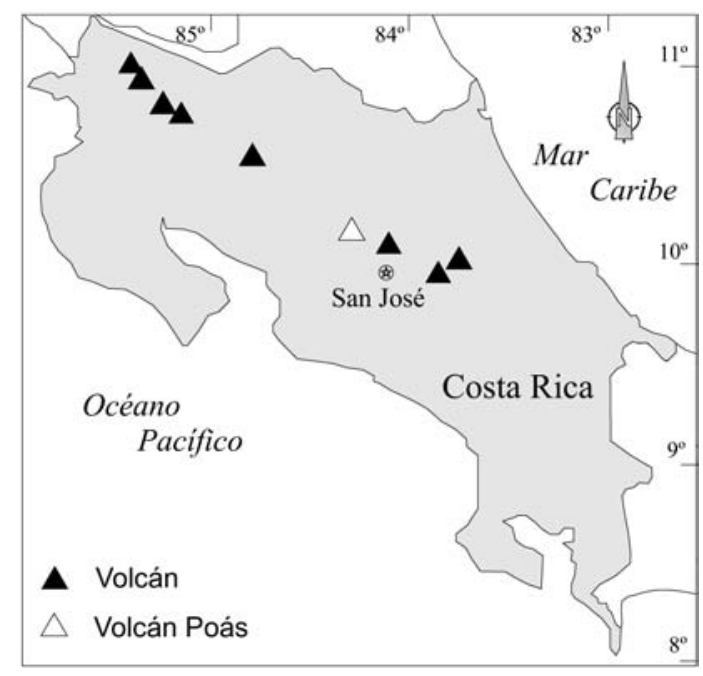

Fig. 1. Localización del volcán Poás en Costa Rica 
Botos (2708 m s.n.m.), localizado al suroeste del cráter activo. Presenta una laguna fría de origen netamente pluvial de unos $400 \mathrm{~m}$ de diámetro y de $15 \mathrm{~m}$ de profundidad en su centro (Rymer et al., 2000).

En este artículo se presentan los resultados del estudio detallado sobre la emisión difusa de hidrógeno en el volcán Poás durante el período 2000-2002. El objetivo principal de este trabajo es detectar y delimitar aquellas áreas que presentan emisiones anómalas de hidrógeno, así como evaluar las variaciones temporales en la distribución de las anomalías y su relación con la actividad volcánica. Este estudio podría ser de especial importancia en la mejora y optimización del programa geoquímico de vigilancia volcánica en el Poás, y así afianzar un enfoque multidisciplinar, contribuyendo a fortalecer los esfuerzos destinados a la reducción del riesgo volcánico en el Poás.

\section{METODOLOGÍA}

Durante los años 2000, 2001 y 2002, se realizaron estudios de emisión difusa de $\mathrm{H}_{2}$ en el volcán Poás. El primero de ellos se realizó entre el 14 de abril y el 4 de mayo del 2000, procediendo a la toma de 176 muestras de gas del suelo en un área de 3,4 km². El segundo estudio se realizó entre el 27 de febrero y el 10 de marzo del 2001, con 254 puntos de medida cubriendo la misma área del año anterior. Por último, entre los días 19 de marzo y 6 de abril del 2002 se materializó la tercera campaña, en la cual se tomaron 295 muestras de gas del suelo, cubriéndose también la misma área de estudio que en años anteriores. Para la selección de los puntos de observación y toma de muestras se tuvo en cuenta las características geológicas y de accesibilidad del área.

En cada punto de observación se recogió una alícuota de gas del suelo mediante una sonda de acero inoxidable de $3 \mathrm{~mm}$ de diámetro interno que se insertaba a $40-50 \mathrm{~cm}$ de profundidad. La toma de muestra de gas se realizó extrayendo $30 \mathrm{~cm}^{3}$ de gas con una jeringuilla hipodérmica y transfiriendo una alícuota de este a un vial sellado de $10 \mathrm{~cm}^{3}$. Para la toma de muestra, se utilizó una variante del método descrito por Hinkle \&
Kilburn (1979), consistente en introducir la muestra de gas en un vial sellado y llenado previamente de agua, de forma que el gas desplaza el agua contenida en el vial. Con este método evitamos que los gases más ligeros se escapen a través de las paredes del tapón del vial debido a la cámara de agua que queda en el interior después de introducir la muestra. Asimismo, en cada punto de observación se determinó la temperatura del suelo a $20 \mathrm{~cm}$ de profundidad.

El análisis de la concentración de $\mathrm{H}_{2}$ en los viales se realizó diariamente en el laboratorio de campo con un microcromatógrafo de gases VARIAN modelo 2002P. Para la determinación del contenido de $\mathrm{H}_{2}$ en el gas del suelo se empleó una columna MolSieve $5 \AA$ de $20 \mathrm{~m}$ de longitud, un detector de conductividad térmica y argón $(99,999 \%)$ como gas portador. Las temperaturas de la columna y del inyector fueron de 60 y $30^{\circ} \mathrm{C}$, respectivamente, mientras que el tiempo de inyección de la muestra fue de 20 milisegundos.

\section{RESULTADOS Y DISCUSIÓN}

En el Cuadro 1 se presenta un resumen de una estadística descriptiva simple de los resultados correspondientes al análisis de las muestras de gas del suelo recogidas en el volcán Poás. Los rangos de concentración de $\mathrm{H}_{2}$ observados fueron de 0,6 a 4,1 ppmV en el año 2000, 0,4 a 1910 ppmV en el año 2001, y 0,8 a 7059 ppmV en el año 2002. Con el objeto de diferenciar la existencia de distintas poblaciones geoquímicas entre el total de las observaciones, se realizó un análisis estadístico-gráfico de Sinclair (1974) para los datos de cada una de las campañas. La distribución log-normal de los valores de concentración de $\mathrm{H}_{2}$ obtenidos en el 2000 reflejó la existencia de una población geoquímica, mientras que los análisis estadístico-gráfico de los datos correspondientes a los años 2001 y 2002, mostraron dos distribuciones normales solapadas que corresponden a las poblaciones de fondo y anómala (Cuadro 2). Los valores medios del contenido de $\mathrm{H}_{2}$ observados para las poblaciones geoquímicas anómalas fueron de 15 y 338 ppmV para 2001 y 2002, respectivamente. La existencia de una población 
Cuadro 1

Estadística descriptiva de los parámetros físico-químicos del volcán Poás

\begin{tabular}{|c|c|c|c|c|c|c|c|c|c|}
\hline & \multicolumn{3}{|c|}{2000} & \multicolumn{3}{|c|}{2001} & \multicolumn{3}{|c|}{2002} \\
\hline & Mín. & Máx. & Med. & Mín. & Máx. & Med. & Mín. & Máx. & Med. \\
\hline $\mathrm{He}(\mathrm{ppmV})$ & 4,2 & 6,3 & 5,6 & 4,4 & 6,0 & 5,5 & 4,8 & 6,1 & 5,8 \\
\hline $\mathrm{H}_{2}(\mathrm{ppmV})$ & 0,6 & 4,1 & 0,7 & 0,4 & 1910 & 15,1 & 0,8 & 7059 & 80 \\
\hline $\mathrm{CH}_{4}(\mathrm{ppmV})$ & 0,9 & 5671 & 47 & 0,4 & 3667 & 748 & 1,1 & 47524 & 6336 \\
\hline $\mathrm{O}_{2}(\%)$ & 20,7 & 21,5 & 19,5 & 12,3 & 23,2 & 21,0 & 0,3 & 21,5 & 20,1 \\
\hline $\mathrm{N}_{2}(\%)$ & 78,1 & 80,6 & 79,6 & 41,7 & 80,5 & 78,0 & 7,4 & 81,7 & 77,6 \\
\hline $\mathrm{CO}_{2}(\%)$ & 0,03 & 58,4 & 1,5 & 0,03 & 53,9 & 14,9 & 0,03 & 80,9 & 21,9 \\
\hline $\mathrm{T}\left({ }^{\circ} \mathrm{C}\right)$ & 7,2 & 92,7 & 13,5 & 0,2 & 92,0 & 13,6 & 7,7 & 144 & 17,6 \\
\hline
\end{tabular}

Cuadro 2

Valores medios de las poblaciones geoquímicas obtenidos mediante el análisis estadístico gráfico de Sinclair (1974)

\begin{tabular}{lccc}
\hline & Población I & Población II & Población III \\
\hline 2000 & 0,6 & 0,7 & n.d. \\
2001 & 0,7 & 1,9 & 15 \\
2002 & 4,1 & 18 & 338 \\
\hline
\end{tabular}

n.d. valor no detectable

anómala en los valores de $\mathrm{H}_{2}$ en el gas del suelo, sugiere una perturbación de origen profundo en el proceso de desgasificación en el ambiente superficial del Poás.

Con la finalidad de evaluar la distribución espacial del contenido de $\mathrm{H}_{2}$ en el gas del suelo del Poás, se construyeron mapas de contorno de la concentración de $\mathrm{H}_{2}$ con el programa SURFER, versión 7.0. Los mapas de contorno se elaboraron de acuerdo con el método estadístico de interpolación "Krigging" aplicando un modelo de variograma experimental lineal como técnica de interpolación (Journel, 1988; Cressie, 1990). En estos mapas se observa que la mayor parte del área de estudio reflejó contenidos de $\mathrm{H}_{2}$ en el suelo relativamente bajos, similares al valor medio estimado para las poblaciones geoquímicas de fondo de cada campaña $(0,6 \mathrm{ppmV}$ para $2000,0,7 \mathrm{ppmV}$ para 2001 y 4,1 ppmV para 2002) y ligeramente superiores al contenido de $\mathrm{H}_{2}$ en el aire atmosférico.

En la campaña del 2000, los mayores niveles de emisión difusa de $\mathrm{H}_{2}$ se localizaron en la zona sureste del área de estudio, donde se alcanzaron concentraciones de 4,4 ppmV de $\mathrm{H}_{2}$. Dentro del cráter principal, los valores más altos de $\mathrm{H}_{2}$ en el gas del suelo se detectaron en el domo y en el sector este del cráter (>1,0 ppmV) (Fig. 2). En la campaña del 2001, los mayores niveles de desgasificación difusa de $\mathrm{H}_{2}$ se observaron en la pared este del cráter principal, donde tiene lugar una intensa actividad fumarólica, alcanzándose concentraciones de 512 ppmV de $\mathrm{H}_{2}$ (Fig. 3). Igualmente, se identificaron contenidos altos de $\mathrm{H}_{2}$ en el gas del suelo de hasta $128 \mathrm{ppmV}$ al sur del domo y dentro del cráter principal. En el área del domo, los valores de concentración de $\mathrm{H}_{2}$ en el gas del suelo, experimentaron un incremento significativo con respecto a los datos observados en la campaña del año 2000, pasándose de concentraciones de $\mathrm{H}_{2}$ de $1,0 \mathrm{ppmV}$ a 32 ppmV. En el año 2002, la actividad fumarólica localizada en la pared este del cráter principal se intensificó con respecto a la existente en los años 2000 y 2001, registrándose en esta zona valores de concentración de $\mathrm{H}_{2}$ en el gas del suelo superiores a $1000 \mathrm{ppmV}$, los cuales duplican los observados en la campaña del año 2001 en esta misma área (Fig. 4). Los mayores valores medidos de concentración de $\mathrm{H}_{2}$ en el gas del suelo para la campaña 

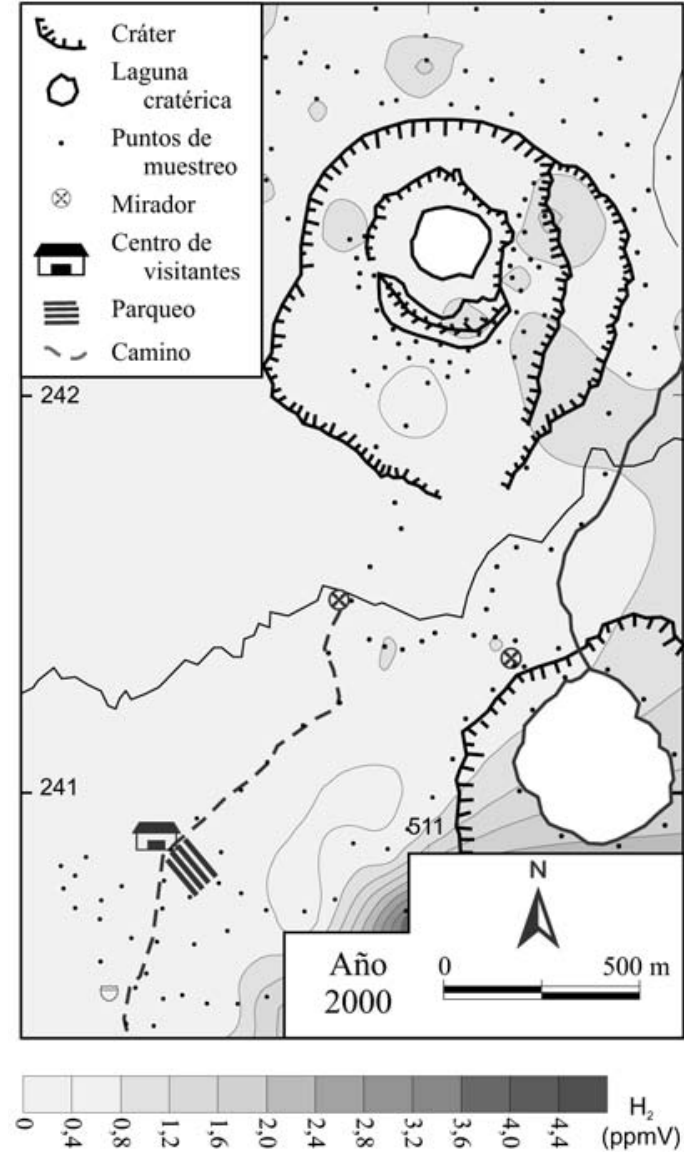

Fig. 2. Distribución espacial de la concentración de hidrógeno en el ambiente superficial del suelo del volcán Poás, Costa Rica (abril 2000).

del 2002 (> 2000 ppmV) se localizaron en la terraza situada en la pared este del cráter principal. Estas fumarolas presentaron un incremento paulatino en su actividad a partir del año 2000. También se midieron contenidos relativamente altos de $\mathrm{H}_{2}$ (> $500 \mathrm{ppmV}$ ) en el domo durante esta campaña de 2002. Los resultados obtenidos evidencian un aumento en la emisión difusa de $\mathrm{H}_{2}$ en el año 2002, con respecto a los años 2000 y 2001.

Los trabajos de emisión de gases y volátiles en suelos de áreas volcánicas realizados por Bertrami et al. (1990) en el volcán Sabatini, Italia, y por Hernández et al. (2000) en las Cañadas del Teide, Islas Canarias, España, hacen referencia a sistemas volcánicos poco activos con niveles de concentración de $\mathrm{H}_{2}$ en el gas del

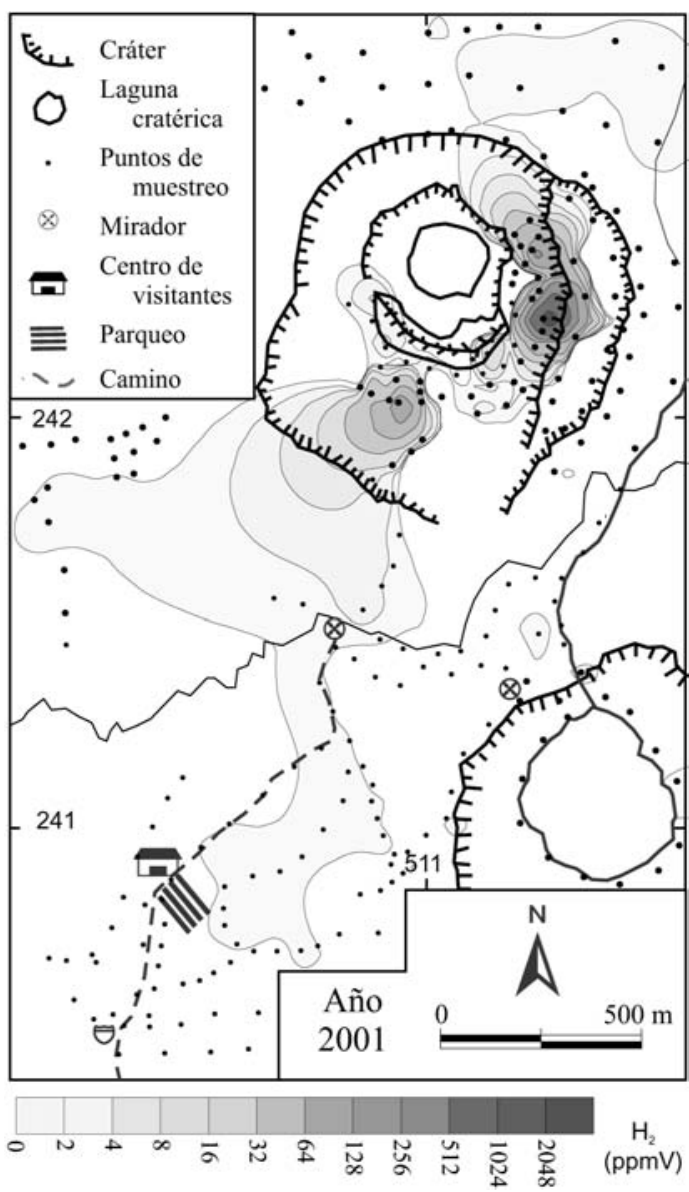

Fig. 3. Distribución espacial de la concentración de hidrógeno en el ambiente superficial del suelo del volcán Poás, Costa Rica (marzo 2001).

suelo inferiores a los observados en el volcán Poás durante los años 2001 y 2002. Bertrami et al. (1990), midieron concentraciones de $\mathrm{H}_{2}$ en el gas del suelo entre 5 y 42 ppmV, con sólo un $10 \%$ del total de las observaciones por encima del límite de detección del instrumento. Hernández et al. (2000), midieron valores de concentración de $\mathrm{H}_{2}$ en el gas del suelo entre 0,5 y 620 ppmV. Los máximos valores en el volcán Poás durante las campañas de 2001 y 2002 fueron de 1910 y 7059 ppmV, respectivamente. Estos contenidos de $\mathrm{H}_{2}$ en los suelos del volcán Poás son dos órdenes de magnitud superior a los observados en el Sabatini y un orden superior a los medidos en las Cañadas del Teide. Esta diferencia en el contenido de $\mathrm{H}_{2}$ en el gas del suelo pudiera ser consecuencia 


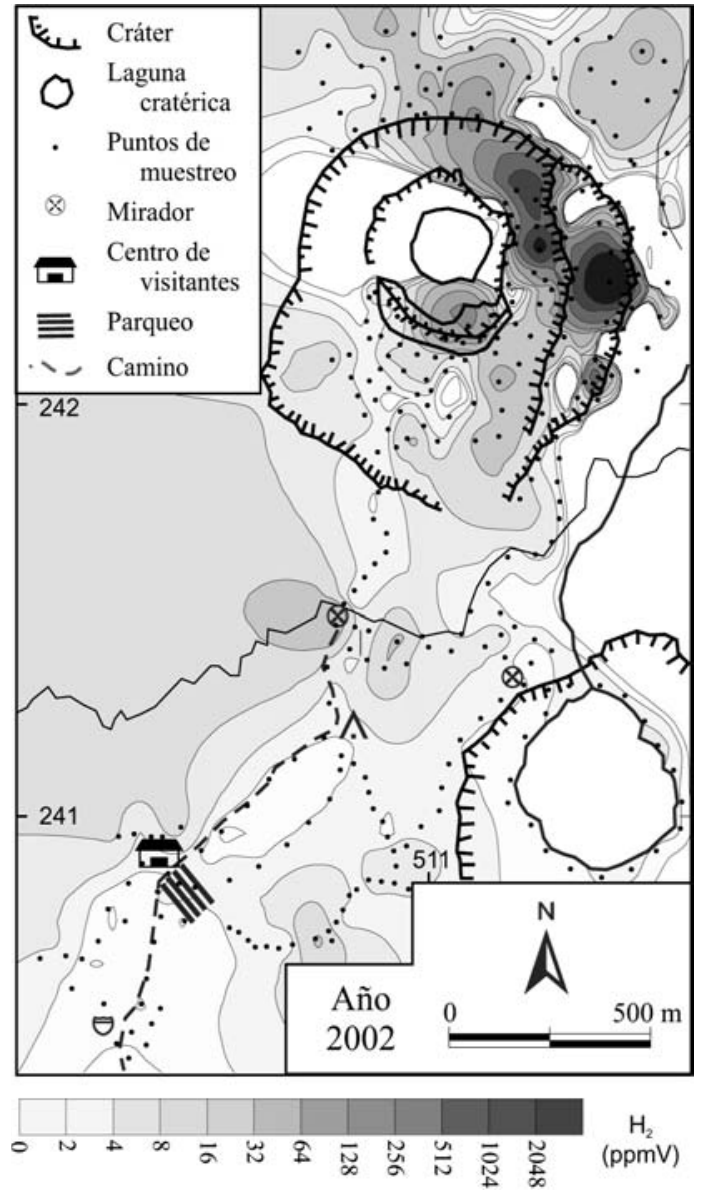

Fig. 4. Distribución espacial de la concentración de hidrógeno en el ambiente superficial del suelo del volcán Poás, Costa Rica (marzo 2002).

del nivel de actividad volcánica que presentaba cada uno de estos sistemas en el momento del estudio. En los casos de Sabatini y Cañadas del Teide, ambos sistemas presentaban un comportamiento estable en su actividad volcánica durante el estudio, mientras que en el caso del Poás se observa un incremento en la actividad de las fumarolas existente en el interior del cráter, así como cambios en la composición química de los condensados de estas y un aumento de la desgasificación, además de la apertura de nuevas ventanas de desgasificación (Fernández et al., 2003) que podrían implicar un cambio en profundidad del sistema volcánico-hidrotermal del Poás.
La temperatura del suelo fue medida también en cada uno de los puntos de muestreo a 20 $\mathrm{cm}$ de profundidad durante las campañas del 2000, 2001 y 2002. Las temperaturas del suelo medidas en la campaña del 2000 variaron entre 7,2 y $92,7^{\circ} \mathrm{C}$, mientras que para las campañas del 2001 y 2002 se observó una variación de las temperaturas entre los 8,3 y $92^{\circ} \mathrm{C}$ y entre los 7,7 y $144^{\circ} \mathrm{C}$, respectivamente. La mayor parte del área de estudio presentó temperaturas del suelo inferiores a $\operatorname{los} 20^{\circ} \mathrm{C}$ para las tres campañas, localizándose las principales anomalías $\left(\mathrm{T}>40^{\circ} \mathrm{C}\right)$ dentro del cráter principal y particularmente donde se encuentra la mayor actividad fumarólica. En el año 2000, la principal anomalía térmica $\left(70^{\circ} \mathrm{C}\right)$ se localizó en el domo, donde estaba concentrada la mayor parte de la actividad fumarólica (Fig. 5a). Durante el año 2001 se produjo un aumento de la temperatura del suelo en el sector este de la pared del cráter principal, así como una disminución de las temperaturas del suelo medidas en el domo, donde se midieron temperaturas aproximadamente $10^{\circ} \mathrm{C}$ por debajo a las medidas el año anterior en esa misma área (Fig. 5b). Durante la campaña de 2002, los valores más altos de temperatura del suelo se midieron nuevamente en la pared este del cráter principal, donde la actividad fumarólica aumentó notablemente con respecto a los años 2000 y 2001, llegándose a medir temperaturas de hasta $140^{\circ} \mathrm{C}$. El área de fumarolas que comenzó a aparecer en el 2000, localizada en la plataforma este del cráter, se incrementó tanto en extensión como en intensidad durante el año 2002, llegándose a medir temperaturas del suelo en torno a los $75^{\circ} \mathrm{C}$, valores similares a los observados en el domo durante la campaña del año 2001 (Fig. 5c).

La distribución espacial de las anomalías de $\mathrm{H}_{2}$ en el gas del suelo observadas durante este periodo de tres años están bien correlacionadas con anomalías de otros gases estudiados, y especialmente con las de flujo de dióxido de carbono (Melián et al., 2001). Los valores medidos de concentración de $\mathrm{H}_{2}$ y de temperatura en el suelo durante las campañas 2001 y 2002 muestran que las zonas de emisión difusa anómala de $\mathrm{H}_{2}$ están caracterizadas por temperaturas del suelo relativamente altas $\left(\mathrm{T}>60^{\circ} \mathrm{C}\right)$, mientras que en 

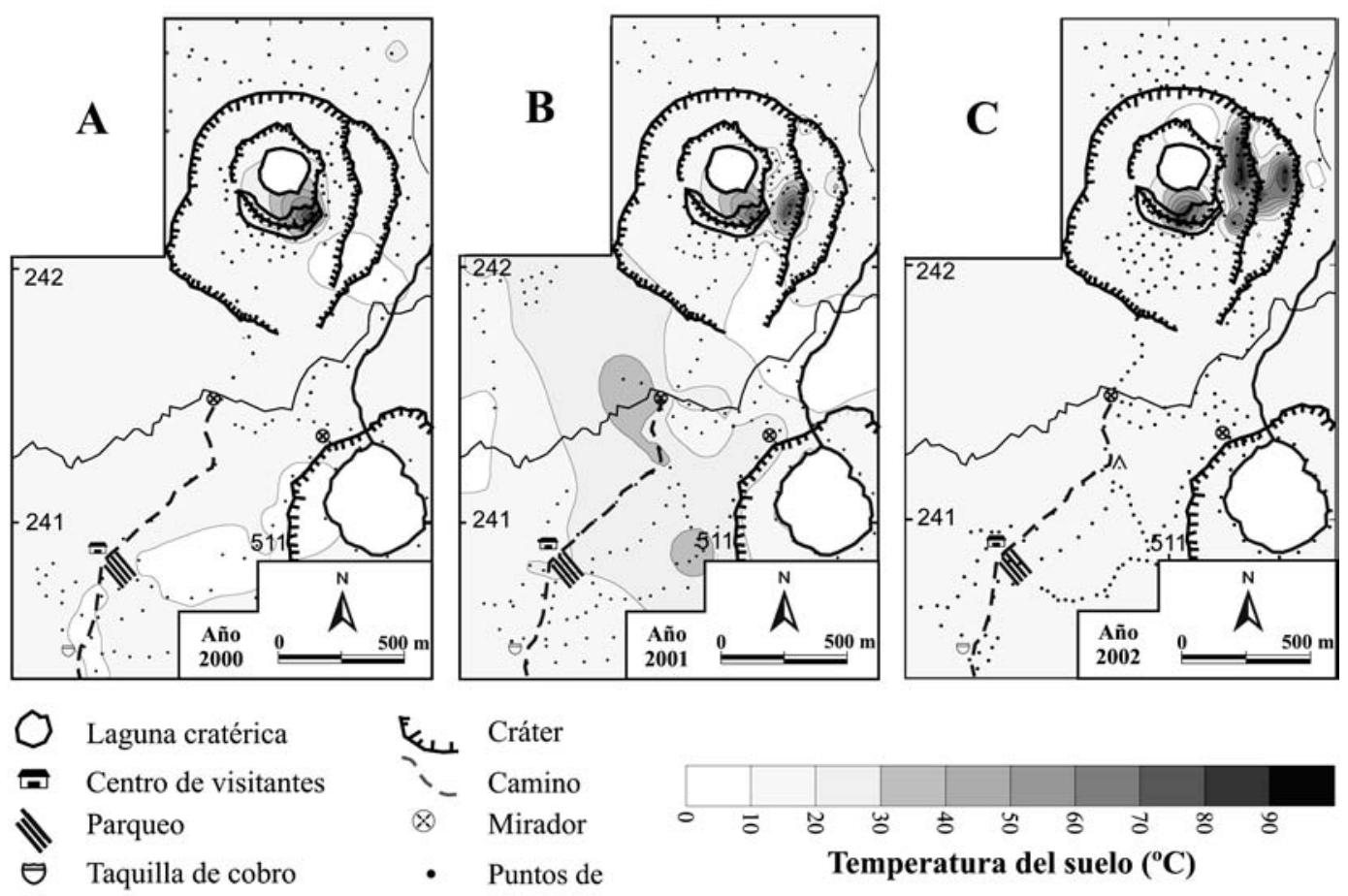

A Estación geoquímica
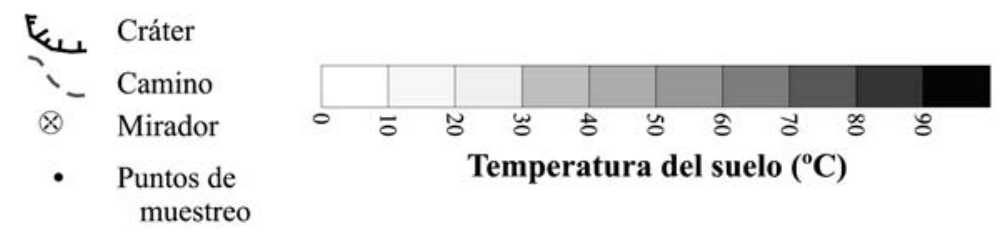

Fig. 5. Distribución espacial de la temperatura del suelo a una profundidad de $20 \mathrm{~cm}$ en el volcán Poás en los años (A) 2000, (B) 2001 y (C) 2002.

las zonas que presentaron bajas concentraciones de $\mathrm{H}_{2}$, la temperatura del suelo es relativamente baja $\left(\mathrm{T}<20^{\circ} \mathrm{C}\right)$. En estas zonas donde se detectaron altas concentraciones de $\mathrm{H}_{2}$ en el gas del suelo, se identificaron también valores anómalos de temperatura del suelo, gradiente de presión y emisión difusa de gases (Melián et al., 2002).

Los coeficientes de correlación entre las concentraciones de $\mathrm{H}_{2}$ y $\mathrm{CO}_{2}$ en el gas del suelo, así como la temperatura del suelo se presentan en el Cuadro 3. Para el año 2000, el coeficiente de correlación entre la concentración de $\mathrm{H}_{2} \mathrm{y} \mathrm{CO}_{2}$ en el gas del suelo es poco significativo $(\mathrm{r}=$ $0,119)$, mientras que para el 2001 y 2002, este coeficiente aumenta notablemente, llegándose a valores de $r=0,473$ y $r=0,726$, respectivamente. Este mismo comportamiento se presenta para las correlaciones entre la concentración de $\mathrm{H}_{2} \mathrm{y}$ la temperatura del suelo con valores de $\mathrm{r}$ en notable ascenso, pasando de 0,011 en el 2000 a 0,497 y 0,416 en el 2001 y 2002, respectivamente. La correlación entre la concentración de $\mathrm{CO}_{2}$ y la temperatura del suelo presenta la misma tendencia, con valores de $r$ muy bajos para el año 2000 $(\mathrm{r}=0,029)$ y aumentando progresivamente en el 2001 y 2002 ( $r=0,375$ y r $=0,511$, respectivamente). La correlación positiva entre la temperatura del suelo y los valores de concentración de $\mathrm{H}_{2}$ en el ambiente superficial sugiere que una parte de la energía procedente de la cámara magmática somera situada a $500 \mathrm{~m}$ de profundidad (Rowe et al., 1995), es utilizada para calentar el agua de origen meteórico. Esta agua meteórica penetra por las fisuras, favoreciendo que parte del $\mathrm{H}_{2}$ emitido por el Poás a la atmósfera sea producido por reacciones redox a alta temperatura entre esta y los minerales máficos presentes mayoritariamente en las rocas basálticas que forman el edificio volcánico. Esta correlación también ratifica la existencia de zonas de alto flujo de calor que reflejan una mayor permeabilidad en profundidad. 
Cuadro 3

Coeficientes de correlación entre la concentración de hidrógeno, dióxido de carbono y la temperatura del suelo

\begin{tabular}{lllllll}
\hline & $\begin{array}{c}\mathrm{H}_{2} \\
(\mathrm{ppmV})\end{array}$ & $\begin{array}{c}\mathrm{CO}_{2} \\
(\mathrm{ppmV})\end{array}$ & $\begin{array}{c}\mathrm{H}_{2} \\
(\mathrm{ppmV})\end{array}$ & $\begin{array}{c}\mathrm{CO}_{2} \\
(\mathrm{ppmV})\end{array}$ & $\begin{array}{c}\mathrm{H}_{2} \\
(\mathrm{ppmV})\end{array}$ & $\begin{array}{c}\mathrm{CO}_{2} \\
(\mathrm{ppmV})\end{array}$ \\
\hline $\mathrm{H}_{2}(\mathrm{ppmV})$ & 1 & & 1 & & 1 & \\
$\mathrm{CO}_{2}(\mathrm{ppmV})$ & 0,119 & 1 & 0,473 & 1 & 0,726 & 1 \\
$\mathrm{~T}\left({ }^{\circ} \mathrm{C}\right)$ & 0,011 & 0,029 & 0,497 & 0,375 & 0,416 & 0,511 \\
\hline
\end{tabular}

Las variaciones temporales observadas en la emisión difusa de $\mathrm{H}_{2}$ y $\mathrm{CO}_{2}$ (Melián et al., 2001, 2002), así como en la temperatura del suelo en el Poás y, la buena correlación existente entre ellas, sugiere que el $\mathrm{H}_{2}$ no se produce solamente por la reacción entre el agua meteórica y los minerales presentes en profundidad, sino que también existen cambios en las condiciones termodinámicas del sistema hidrotermal del volcán Poás que originan estos incrementos.

Los procesos que tienen lugar en profundidad en un sistema volcánico pueden dar origen a un aumento de la emisión de gases y volátiles. Estos procesos son muy diversos. Para explicar el incremento en la emisión difusa de $\mathrm{H}_{2}$ observado en el periodo 2000-2002 en el volcán Poás, se plantean dos posibles procesos que tienen lugar en profundidad. Un primer proceso podría consistir en la desestabilización del sistema originada por el movimiento de fallas adyacentes al volcán. Un segundo proceso implicaría una pequeña intrusión de magma que no llega a superficie.

La primera hipótesis plantea la existencia de cambios de esfuerzos locales ocasionados por la inusual actividad tectónica en el volcán originada a comienzos de julio de 1999. El 18 de julio de ese mismo año, se registró un sismo de M 3,2 localizado al sur del cráter, continuando la actividad tectónica hasta el año 2000, con un número menor de sismos, pero de mayor intensidad. Esta sismicidad estaría relacionada con el movimiento de fallas adyacentes al volcán (Mora et al., este volumen). La actividad tectónica registrada podría haber aumentado la fracturación en la zona, induciendo cambios en la permeabilidad del sistema que se manifestaron en una mayor desgasificación en superficie.

La formación de una pequeña intrusión de magma que no llega a superficie es la segunda hipótesis planteada. Para la formación de una intrusión, es necesario que la presión interna de la cámara sea superior a la suma de la presión litostática más la resistencia a la ruptura de la roca encajante, lo que provoca la fracturación de las paredes de la cámara y la salida de una pequeña cantidad de material. La cantidad de material que sale dependerá de la diferencia entre la presión de la cámara magmática y la presión litostática. Este aumento de presión puede producirse de dos formas distintas: (a) por un aporte de nuevo magma poco desgasificado a la cámara magmática somera del Poás, o (b) por una diferenciación del magma dentro de la cámara, debido a un proceso de cristalización fraccionada. Este aumento de presión en el interior de la cámara es el que favorece la salida de gases a la superficie. El emplazamiento de esta pequeña intrusión se localizaría entre la zona este de la laguna caliente y la pared este del cráter, según la sismicidad detectada en la zona entre finales de 1999 y principios de 2000 (Fernández et al., 2003). Las dimensiones del área de emplazamiento sugieren que esta pequeña intrusión de magma se puede considerar como un dique que no llega a superficie. Este tipo de inyecciones desde la cámara magmática son muy comunes en periodos de inactividad en los volcanes. Esta observación es propuesta por Newhall \& Dzurisin (1988), después de estudiar varias intrusiones en volcanes no activos, que no se asociaron a periodos eruptivos. 
Una combinación de ambas hipótesis no habría que descartarla para explicar el aumento en la emisión de gases, ya que por lo general los procesos que se desarrollan en profundidad son complejos y no ocurren independientemente unos de otros. La inestabilidad del sistema ocasionada por la actividad sísmica en julio de 1999, pudo generar un cambio en la presión interna de la cámara produciendo una pequeña intrusión en la zona este del cráter principal.

La forma de discernir cuál de las hipótesis explicaría mejor el aumento en la emisión de $\mathrm{H}_{2}$ en el gas del suelo del volcán Poás implicaría la realización de estudios isotópicos de este gas del suelo para evaluar el origen del hidrógeno, además de compaginar técnicas geofísicas como gravimetría y sísmica. Asimismo, una ampliación del número de estaciones sísmicas en la red del Poás sería de gran ayuda para entender los procesos que están ocurriendo en profundidad. Estos datos, combinados con los estudios de desgasificación difusa que se están realizando anualmente en el volcán Poás, y la información geoquímica de los gases y condensados fumarólicos, nos permitirían tener una visión más amplia de los procesos que están teniendo lugar en el sistema volcánico-hidrotermal del Poás.

\section{CONCLUSIONES}

Los resultados obtenidos en este estudio reflejan que se ha producido un aumento significativo del la emisión difusa de $\mathrm{H}_{2}$ en el gas del suelo del volcán Poás. Los mayores incrementos de $\mathrm{H}_{2}$ se han detectado en el sector este del cráter principal, donde se ha intensificado la actividad fumarólica. La buena correlación existente entre la emisión difusa de $\mathrm{H}_{2}$ y $\mathrm{CO}_{2}$, así como de la temperatura del suelo, reflejan la existencia de zonas de mayor permeabilidad en el área de estudio que facilitan el escape de gases de origen profundo al ambiente superficial. Este aumento en los niveles de emisión difusa de $\mathrm{H}_{2}$ pudiera estar relacionado con un cambio en profundidad del sistema volcánico-hidrotermal del volcán Poás, reflejándose la utilidad de este tipo de estudios para mejorar y optimizar la sistemática de detección de señales de alerta temprana sobre posibles crisis volcánicas que pudieran ocurrir en el Poás.

\section{AGRADECIMIENTOS}

Agradecemos a Juan Dobles y a todos los guardas del Parque Nacional Volcán Poás por su hospitalidad y amabilidad durante nuestra estancia en las instalaciones del parque. También queremos agradecer al grupo COVIRENAS, a su director Carlos Cordero y a Juan Carlos Mesa por su ayuda desinteresada en las labores de campo. Este trabajo se ha podido realizar gracias al Cabildo Insular de Tenerife y CajaCanarias (Tenerife, España).

\section{REFERENCIAS}

ALLARD, P., CARBONELLE, J., DAJLEVIC, D., BRONCE, J., MOREL, P., ROBE, M., MAURENADS, J., FAIVRE-PIERRET, R., MARTIN, D., SABROUX, J., \& ZETTWOOG, P., 1991: Eruptive and diffuse emissions of $\mathrm{CO}_{2}$ from Mount Etna. - Nature, 351: 387-391.

BERTRAMI, R., BUONASORTE, G., CECCARELLI, A., LOMBARDI, S., PIERI, S. \& SCANDIFFIO, G., 1990: Soil gas in geothermal prospecting: two case histories (Sabatini Volcanoes and Alban Hills, Latium, Central Italy). - J. Geophys. Res. 95: 2147521481.

BROWN, G., DOWDEN, J., KAPADIA, P., STEVENSON, D., BARQUERO, J. \& MORALES, L., 1989: Energy budget analysis for Poás crater lake: implications for predicting volcanic activity. - Nature, 339: 370-373.

CASADEVALL, T., DOUKAS, M., NEAL, C., MCGIMSEY, R. \& GARDNER, C., 1994: Emission rates of sulphur dioxide and carbon dioxide from Redoubt Volcano, Alaska during the 1989-1990 eruptions. - J. Volcanol. Geotherm. Res. 62: 519-530.

CASERTANO, L., BORGIA, A., CIGOLINI, C., MORALES, L., MONTERO, W., GÓMEZ, M. \& FERNÁNDEZ, J.F., 1985: Investigaciones geofísicas y características geoquímicas de las aguas hidrotermales: Volcán Poás, Costa Rica. - Geofís. Int. 24(2): 315-332. 
CASERTANO, L., BORGIA, A., CIGOLINI, C., MORALES, L., GÓMEZ, M. \& FERNÁNDEZ, J.F., 1987: An integrated dynamic model for the volcanic activity at Poás Volcano, Costa Rica. - Bull. Volcanol. 49: 588-598.

CRESSIE, N., 1990: The origins of Krigging. - Math. Geol. 22: 239 .

FERNÁNDEZ, E., DUARTE, E., SÁENZ, W., MALAVASSI, E., BARBOZA, V., MARTÍNEZ, M. \& VALDEZ, J., 2003: Volcanic gas condensates from Poás volcano 1999-2000. - [res.] Eighth Field Workshop on Volcanic Gases. IAVCEI \& CCVG.

GERLACH, T. \& GRAEBER, E., 1985: Volatile budget of Kilauea volcano. - Nature, 313: 273-277.

GIGGENBACH, W., 1996: Chemical composition of volcanic gases. - En: SCARPA, R. \& TILLING, R. (eds.): Monitoring and mitigation of volcanic hazards. Springer, Berlin: 221-256.

HERNÁNDEZ, P., PÉREZ, N., SALAZAR, J., SATO, M., NOTSU, K. \& WAKITA, H., 2000: Soil gas $\mathrm{CO}_{2}$, $\mathrm{CH}_{4}$ and $\mathrm{H}_{2}$ distribution in and around Las Cañadas caldera, Tenerife, Canary Islands, Spain. - J. Volcanol. Geotherm. Res. 103: 425-438.

HERNÁNDEZ, P., SALAZAR, J., SHIMOIKE, Y., MORI, T., NOTSU, K. \& PÉREZ, N., 2001a: Diffuse emission of $\mathrm{CO}_{2}$ from Miyakejima volcano, Japan. Chem. Geol. 177: 175-185.

HERNÁNDEZ, P., NOTSU, K., SALAZAR, J., MORI, T., NATALE, G., OKADA, H., VIRGILI, G., SHIMOIKE, Y., SATO, M. \& PÉREZ, N. 2001b: Carbon dioxide degassing by advective flow from Usu volcano, Japan. - Science, 292: 83-86.

HINKLE, M. \& KILBURN, J., 1979: The use of vacutainer tube for collection of soil sample for helium analysis.- U. S. Geol. Surv. Open File Rep. 79: 1441.

HIRABAYASHI, J., OSSAKA, J. \& OZAWA, T., 1986: Geochemical study on volcanic gases at Sakurajima volcano, Japan. - J. Geophys. Res. 91: 12167-12176.

JOURNEL, A., 1988: Principles of Environment Sampling. En: KEITH, L. (ed.): Am. Chem. Soc.: 45-72.

LOMBARDI, S. \& REIMER, G., 1990: Radon and helium in soil gases in the Phlegraean Fields, Central Italy. Geophys. Res. Lett. 17(6): 849-852.

MCGEE, K. \& GERLACH, T., 1998: Airborne volcanic plume measurements using a FTIR spectrometer, Kilauea volcano, Hawaii. - Geophys. Res. Lett. 25(5): 615-618.
MELIÁN, G., GALINDO, I., SALAZAR, J., HERNÁNDEZ, P., PÉREZ, N., RAMÍREZ, C., FERNÁNDEZ, M. \& NOTSU, K., 2001: Spatial and secular variations of diffuse $\mathrm{CO}_{2}$ degassing from Poás volcano, Costa Rica, Central America. - [res.] EOS, Transactions, AGU, 82: F1332.

MELIÁN, G., GALINDO, I., SALAZAR, J., PÉREZ, N., HERNÁNDEZ, P., FERNÁNDEZ, M., RAMÍREZ, C., MORA, R. \& ALVARADO, G., 2002: Hydrogen emission from Poás volcano, Costa Rica, Central America: a premonitory geochemical signature of volcanic unrest? - [res.] EOS, Transactions, AGU, 83: F1489.

MORA, R., RAMÍREZ C. \& FERNÁNDEZ M., 2004: La actividad de los volcanes de la Cordillera Central, Costa Rica, entre 1998-2002. - Rev. Geol. Amér. Central (este volumen).

NEWHALL, C. \& DZURISIN, D., 1988: Historical unrest at large calderas of the world. - US Geol. Surv. Bull. 1855: 1-1108.

PAN, V., HOLLOWAY, J. \& HERVIG, R., 1991: The pressure and temperature dependence of carbon dioxide solubility in tholeiitic basalts melts. - Geochim. Cosmochim. Acta, 55: 1587-1595.

PÉREZ, N., WAKITA, H., LOLOK, D., PATIA, H., TALAI, B. \& MCKEE, C., 1996: Anomalous soil gas $\mathrm{CO}_{2}$ concentrations and relation to seismic activity at $\mathrm{Ra}-$ baul caldera, Papua New Guinea. - Geogaceta, 20: 1000-1003.

ROWE, G., BRANTLEY, S., FERNÁNDEZ, M., FERNÁNDEZ, J.F., BARQUERO, J. \& BORGIA, A., 1992a: Fluid-volcano interacting in an active stratovolcano: The crater lake system of Poás Volcano, Costa Rica. - J. Volcanol. Geotherm. Res. 49: 23-51.

ROWE, G., OHSAWA, S., TAKANO, B., BRANTLEY, S., FERNÁNDEZ, J.F. \& BARQUERO, J., 1992b: Using crater lake chemistry to predict volcanic activity at Poás Volcano, Costa Rica. - Bull. Volcanol. 54: 494-503.

ROWE, G., BRANTLEY, S., FERNÁNDEZ, J. \& BORGIA, A., 1995: The chemical and hydrologic structure of Poás Volcano, Costa Rica. - J. Volcanol. Geotherm. Res. 64: 233-267.

RYMER, H., CASSIDY, J., LOCKE, C., BARBOZA, M., BARQUERO, J., BRENES, J. \& VAN DER LAAT, R., 2000: Geophysical studies of the recent 15-year eruptive cycle at Poás Volcano, Costa Rica. - J. Volcanol. Geotherm. Res. 97: 425-442. 
SALAZAR, J., HERNÁNDEZ, P., PÉREZ, N., MELIÁN, G., ÁlVAREZ J., SEGURA, F. \& NOTSU, K., 2001: Diffuse emission of carbon dioxide from Cerro $\mathrm{Ne}$ gro Volcano, Nicaragua. - Geophys. Res. Lett. 28: 4275-4278.

SANO, Y., NOTSU, K., ISHIBASHI, J., IGARASHI, G. \& WAKITA, H., 1991: Secular variations in helium isotope ratios in an active volcano: Eruption and plug hypothesis. - Earth Planet. Sci. Lett. 107: 95-100.

SATO, M., 1988: Continuous monitoring of hydrogen in volcanic areas: Petrological rationale and early experiments. - Rendiconti della Società Italiani di Mineralogia Petrologia, 43, 1265-1281.

SATO, M. \& MCGEE, F., 1981: Continuous monitoring of hydrogen on the south flank of Mount St. Helens. En: LIPMAN, P. W., MULLIMEAUX, D. R. (eds.):
The 1980 eruption of Mount St. Helens, Washington. - U.S. Geol. Surv. Prof. Pap. 1250: 209-219.

SINCLAIR, A., 1974: Selection of thresholds in geochemical data using probability graphs. - J. Geochem. Explor. 3: $129-149$.

SIMKIN, T. \& SIEBERT L., 1994: Volcanoes of the world [2nd ed.]. - 349 págs. Geoscience Press, Tuscon, Arizona.

STOLPER, E. \& HOLLOWAY, J., 1988: Experimental determination of the solubility of carbon dioxide in molten basalt at low pressure. - Earth Planet. Sci. Lett. 87: 397-408.

WHITE, D. \& WARING, G., 1963: Volcanic emanations. En: CHAPTER K.: Data of geochemistry [6th ed.]. - US Geol. Surv. Prof. Pap. 440-K:1-27. 